\title{
Autonomic and emotion regulation in bereavement and depression
}

\author{
Mary-Frances O'Connor*, John J.B. Allen, Alfred W. Kaszniak \\ Department of Psychology, Building 68, University of Arizona, Tucson, AZ 85721, USA
}

Received 13 December 2000; accepted 1 November 2001

\begin{abstract}
Objectives: Prior research suggests important differences between depression and the depressed feelings experienced in the context of bereavement, despite some overlap. Differences include an increase in restlessness, suggesting underlying physiological differences between the groups. Method: This study examined the level of depressive symptoms, heart rate (HR), and heart rate variability (HRV), and coping style of 10 bereaved, 10 depressed, and 10 control participants. Results: Bereaved participants showed significantly higher HR than either depressed or control participants, while there were no such
\end{abstract}

differences in HRV. Level of depression in the bereaved group correlated negatively with HRV. Additional analyses showed that the use of passive coping had a marginally significant negative correlation with HRV in bereaved subjects. Conclusion: The present data suggest that differences in HR and HRV could be associated with increased cardiovascular fatalities in bereaved individuals, known as the "broken heart phenomenon." These physiological differences have potential implications for both the mental and physical health of the bereaved. (C) 2002 Elsevier Science Inc. All rights reserved.

Keywords: Autonomic; Bereavement; Coping; Depression; Emotion; Heart rate

\section{Introduction}

There may exist both similarities and differences between depression and depressed feelings experienced during bereavement. In the 1970s, Clayton et al. [1-3] reported a distinct pattern of depressive symptoms in bereaved individuals. At 1 month, symptoms included crying, low mood, sleep disturbance (especially initial insomnia), loss of appetite, restlessness, fatigue, poor memory, and loss of interest [4]. At the end of 1 year, many of the vegetative symptoms improved, but insomnia, restlessness, and periodic low mood persisted. The high occurrence (and persistence) of restlessness has been replicated [5-7].

Approximately $50 \%$ of widows meet criteria for major depression sometime during the first year after the death of the spouse [8]. The similarity between the phenomenology of depression and depressive symptoms during bereavement, however, may not reflect similar underlying etiological mechanisms. For example, a treatment study with nortriptyline and interpersonal therapy found that although

\footnotetext{
* Corresponding author. Tel.: +1-520-621-7447; fax: +1-520-621-
}

depressive symptoms decreased with treatment, bereavement intensity was not impacted by either treatment [9].

The present investigation therefore examined psychophysiological profiles of bereavement and depression to address the question of whether similar symptomatology is produced by similar underlying mechanisms. In addition to the previously reviewed differences in restlessness, heart rate (HR) and heart rate variability (HRV) may be important markers in depression and bereavement.

Decreased HRV has been observed in depression [1012], although gender may play an important role [13]. There is some evidence that successful treatment of depression is accompanied by an increase in HRV [14-16]. HRV reflects changes in beat-to-beat intervals, and such variability in the $0.12-0.40 \mathrm{~Hz}$ range is thought to be primarily under vagal control. No studies of HR and HRV in bereaved individuals have been reported.

The "broken heart" phenomenon, or the increased rate of sudden cardiac death in bereaved spouses, suggests that heart health is affected in bereavement [17]. Widows and widowers are more likely to die than their married peers of the same age [18], and these individuals are at greater risk during the first 6 months of widowerhood [19]. Support for the "broken heart" phenomenon comes from data 
demonstrating that $75 \%$ of the increased death rate is due to heart disease [20].

The death of a loved one also gives rise to a secondary set of psychosocial circumstances. These include lack of contact with other people, feelings of isolation [21] and a climate of helplessness, feelings of a lack of control and coping, and depression [21]. Bereavement alone does not appear to increase the risk of illness and death, but rather, bereavement coupled with depression is thought to be the crucial interaction. In addition, depression has been shown to be an independent risk factor for cardiac morbidity and mortality [22-24].

The present study provides an initial exploration of HR and HRV in depressed, bereaved, and control subjects. It was hypothesized that the bereaved group would have higher HRs than the depressed group, based on their symptom profile. It was further hypothesized that depressed, bereaved individuals will show lower HRV than nondepressed, bereaved individuals. The present study also included a measure of coping styles, with the hypothesis that less adaptive coping styles would correlate with lower HRV.

\section{Method}

Thirty participants were recruited: 10 bereaved (brv), 10 depressed (dep), and 10 controls (ctl). Bereavement was defined as having experienced the death of a first- or second-degree family member or close friend. Five participants suffered the loss of a parent, two lost grandfathers, and one each lost a partner, best friend, and sister. The death occurred in the last 2-24 months (mean=5.2). Depression was defined by DSM-IV criteria for major depression that did not have an onset following the death of a loved one, and no other Axis I or Axis II disorders. Exclusion criteria for all groups included a history of heart disease or receipt of prescription cardiac medication, and age greater than 65 . Exclusion criteria for control participants also included having experienced the death of a loved one in the prior 2 years and/or major depression.

All participants were recruited through advertisements. There were no differences between groups in age (mean brv $=32.6 ; \operatorname{dep}=33.7 ; \mathrm{ctl}=33.6)$ or gender $($ brv $=80 \%$ female; dep $=80 \%$ female; ctl $=80 \%$ female). Table 1 provides descriptive data for participants.

Depression symptom severity for the depressed, bereaved, and control groups was assessed with the Beck Depression Inventory (BDI), the Hamilton Rating Scale for Depression (HRSD), and the depression section of the Structured Clinical Interview for the DSM-IV (SCID-I). Other variables included the COPE Scale [25], a 60-item questionnaire developed to assess a variety of coping styles used after a stressful life event.

The electrocardiogram (ECG) was recorded for $5 \mathrm{~min}$ prior to and following the structured interview. ECG was recorded with the $\mathrm{J} \& \mathrm{~J}$ amplifier system, attaching silver-
Table 1

Participant characteristics (standard deviations in parentheses)

\begin{tabular}{llllr}
\hline & Sex & Age & Hamilton-D & SCID \\
\hline Bereaved & $80 \% \mathrm{~F}$ & $32.6(9.41)$ & $18.8(8.02)$ & $50 \%$ \\
Depressed & $80 \% \mathrm{~F}$ & $33.7(8.86)$ & $21.2(2.66)$ & $100 \%$ \\
Control & $80 \% \mathrm{~F}$ & $35.4(14.11)$ & $2.4(1.51)$ & $0 \%$ \\
\hline
\end{tabular}

SCID refers to number of subjects meeting DSM-IV criteria for major depression, exclusive of the bereavement rule-out. In other words, among bereaved subjects, none technically meet criteria for major depressive disorder due to the fact that the depressive symptoms onset following the death of a close loved one. The rate of $50 \%$ is provided descriptively to provide a sense of the proportion of subjects experiencing substantial depressive symptoms in the context of bereavement.

silver chloride electrodes to the left and right upper forearm with Signa Crème electrode conductive cream. The ground was attached to the lower right forearm. No instructions were given on how to breathe. ECG was sampled at $1024 \mathrm{~Hz}$. Ectopic beats and abnormalities were monitored on the screen.

Interbeat interval series were first derived from the raw ECG, using an r-spike detection algorithm, followed by hand screening with correction for artifacts (missed or erroneous beats). The index of HRV primarily under vagal control is the respiratory sinus arrhythmia (RSA) band $(0.12-0.4 \mathrm{~Hz})$. RSA was extracted using Delta-Biometrics, MX Edit software.

\section{Results}

To test for group differences in HR, a Group $\times$ Time (before or after interview) analysis of variance (ANOVA) was used. The bereaved group had a significantly higher HR than either the depressed or normal control groups $[\mathrm{brv}=84.84, \operatorname{dep}=72.32, \operatorname{ctl}=72.90 ; F(2,27)=5.10$, $P<.01]$. There was no significant difference in RSA between the groups $[\mathrm{brv}=6.19, \mathrm{dep}=6.10, \mathrm{ctl}=5.57$; $F(2,27)=0.79, P<.46]$.

Analyses were conducted on the bereaved group separately to determine the correlation between level of depression (using BDI scores) and HRV. Due to incomplete self-report data, for these analyses, the bereaved group contained nine participants. A significant negative correlation was found between depression scores and RSA $(r=-.67, P<.05)$.

The COPE scale clusters into several subscales [25]. Correlational analyses were conducted on four of these subscales to determine whether coping behavior could predict HR and HRV in the bereaved group $(n=9)$. Carver et al. [25] describe "adaptive coping" subscales, which include active coping and acceptance, and "questionable value" coping scales, which include passive coping and disengaging through drug and alcohol use. Table 2 summarizes the results.

These correlations between HR, HRV, and COPE styles are all in the expected direction. The use of more adaptive coping is correlated with higher HRV and a lower HR. The 
Table 2

Correlations between cardiovascular variables and coping subscales

\begin{tabular}{llr}
\hline & \multicolumn{2}{l}{ Autonomic variables } \\
\cline { 2 - 3 } COPE & HR & HRV \\
\hline Acceptance & -.12 & .40 \\
Active & -.24 & .39 \\
Drug use & .18 & -.18 \\
Passive & .37 & $-.62^{\dagger}$ \\
\hline
\end{tabular}

${ }^{\dagger} P<.10$.

use of poor coping is correlated with lower HRV and a higher HR. While none of the correlations reached conventional levels of statistical significance, likely due to low-power, passive coping (including mental and behavioral disengagement and denial) had a trend toward a statistically significant negative correlation with HRV $(r=-.62, P<.08)$.

\section{Discussion}

This exploratory investigation of the psychophysiology of bereaved, depressed, and control individuals showed that bereaved individuals are characterized by a significantly higher HR than either the depressed or the control participants, in the absence of group differences in HRV. In addition, within the bereaved group, individuals with higher levels of depression showed lower levels of HRV. Although based on a small sample, the present study is the first to examine differences in autonomic psychophysiology between depressed and bereaved individuals.

Increased HR and decreased HRV suggest a mechanism underlying the increased rate of heart disease and fatalities seen in bereaved individuals as compared to their married counterparts [17-20]. This study may warrant further larger-scale studies of cardiovascular and emotional function in relation to the health of bereaved individuals, and as a function of the length of time since the bereavement began or the relationship to the deceased individual.

This exploratory and cross-sectional study could not address whether HR or HRV changes during the course of bereavement and depression, and whether they act as a result of interventions designed to assist bereaved individuals. The present results suggest the utility of a longitudinal investigation with interventions focused on individuals' coping styles to determine whether the cardiovascular correlates of depression in bereavement can be normalized.

\section{References}

[1] Clayton PJ, Herjanic M, Murphy GE, Woodruff R. Mourning and depression: their similarities and differences. Can Psychiatr Assoc J 1974;19(3):309-12.
[2] Bornstein PE, Clayton PJ, Halikas JA, Maurice WL, Robins E. The depression of widowhood after thirteen months. Br J Psychiatry 1973;122(570):561-6.

[3] Clayton PJ, Halikas JA, Maurice WL. The bereavement of the widowed. Dis Nerv Syst 1971;32(9):597-604.

[4] Clayton PJ. Bereavement and depression. J Clin Psychiatry 1990; 51:34-40.

[5] Blanchard CG, Blanchard EB, Becker JV. The young widow: depressive symptomatology throughout the grief process. Psychiatry 1976; 39(4):394-9.

[6] Jacobs S, Hansen F, Berkman L, Kasl S, Ostfeld A. Depression of bereavement. Compr Psychiatry 1989;30(3):218-24.

[7] Pasternak RE, Reynolds CF, Miller MD, Fasiczka AL, Prigerson H, Frank E, Kupfer DJ. Spousally bereaved elders with subsyndromal depression: a descriptive analysis and comparison with major depression. Am J Geriatr Psychiatry 1996;4(1):61-8.

[8] Zisook S, Shuchter SR. Depression through the first year after the death of a spouse. Am J Psychiatry 1991;148:1346-52.

[9] Reynolds CF, Miller MD, Pasternak RE, Frank E, Perel JM, Cornes C, Houck PR, Mazumdar S, Dew MA, Kupfer DJ. Treatment of bereavement-related major depressive episodes in later life: a controlled study of acute and continuation treatment with nortriptyline and interpersonal therapy. Am J Psychiatry 1999;156(2):202-8.

[10] Rechlin T, Weis M, Spitzer A, Kaschka WP. Are affective disorders associated with alterations of heart rate variability? J Affect Disord 1994;32(4):271-5.

[11] Rechlin T, Weis M, Kaschka WP. Is diurnal variation of mood associated with parasympathetic activity? J Affect Disord 1995;34(3): $249-55$.

[12] Roose SP, Glassman AH, Dalack GW. Depression, heart disease, and tricyclic antidepressants. J Clin Psychiatry 1989;50:12-6.

[13] Thayer JF, Smith M, Rossy LA, Sollers JJ, Friedman BH. Heart period variability and depressive symptoms: gender difference. Biol Psychiatry 1998;44(4):304-6.

[14] Chambers A, Allen JJB. Predicting treatment response in major depression with respiratory sinus arrhythmia. Poster presentation: Soc Psychophysiol Res, 1999.

[15] Balogh S, Fitzpatrick DF, Hendricks SE, Paige SR. Increases in heart rate variability with successful treatment in patients with major depressive disorder. Psychopharmacol Bull 1993;29(2):201-6.

[16] Khaykin Y, Dorian P, Baker B, Shapiro C, Sandor P, Mironov D, Irvine J, Newman D. Automatic correlates of antidepressant treatment using heart-rate variability analysis. Can J Psychiatry 1998;43(2):183-6.

[17] Stroebe M. The broken heart phenomenon: an examination of the mortality of bereavement. J Community Appl Soc Psychol 1994; 4:47-61.

[18] Kraus AS, Lilienfeld AM. Mortality by marital status. J Chronic Dis 1959;10:207-11.

[19] Young M, Benjamin B, Wallis C. Mortality of widowers. Lancet 1963; 2:454-6.

[20] Parkes CM, Benjamin B, Fitzgerald RG. Broken heart: a statistical study of increased mortality among widowers. Br Med J 1969;1:740-3.

[21] Boman B. Stress and heart disease. In: Fisher S, Reason J, editors. Handbook of life stress, cognition and health. New York, NY: John Wiley \& Sons, 1988. pp. 301-15.

[22] Strik J, Honig A, Maes M. Depression and myocardial infarction: relationship between heart and mind. Prog Neuro-Psychopharmacol Biol Psychiatry 2001;25(4):879-92.

[23] Roose SP. Depression, anxiety, and the cardiovascular system: the psychiatrist's perspective. J Clin Psychiatry 2001;62(18):19-22.

[24] Sher L. Psychological factors, immunity, and heart disease. Psychosomatics 2000;41(4):372-3.

[25] Carver CS, Scheier MF, Weintraub JK. Assessing coping strategies: a theoretically based approach. J Pers Soc Psychol 1989;56(2): $267-83$. 